\title{
Detection of on-aircraft fatigue cracks in a UH-60A planet carrier using spectral averaging
}

\author{
B Van Hecke, J Yoon and D He
}

\begin{abstract}
In this paper, a new method for planet carrier fatigue crack detection is presented. This method begins by time synchronously resampling vibration signals to segment the signals according to shaft crossing times, such that an even number of data points are obtained to compute a spectral average. Then, the result is used to extract features and evaluate numerous condition indicators (Cls) for planet carrier fatigue crack detection. The methodology is validated using data from a number of US Army UH-60A Black Hawk helicopter main transmissions. The results are presented using vibration measurements of faulted and unfaulted transmissions over low-torque levels in on-aircraft conditions.
\end{abstract}

Keywords: fatigue crack detection, vibration, spectral averaging.

\section{Introduction}

The transmission of the UH-60A helicopter, shown in Figures 1 and 2, contains a planetary gear train in the final stage of the main rotor gearbox. In this configuration, torque is transmitted from the central sun gear through the planets to the planet carrier and then from the planet carrier to the main rotor shaft. To ensure the safety of the aircraft and passengers, it is important to have tools to determine the condition of critical components. Although difficult

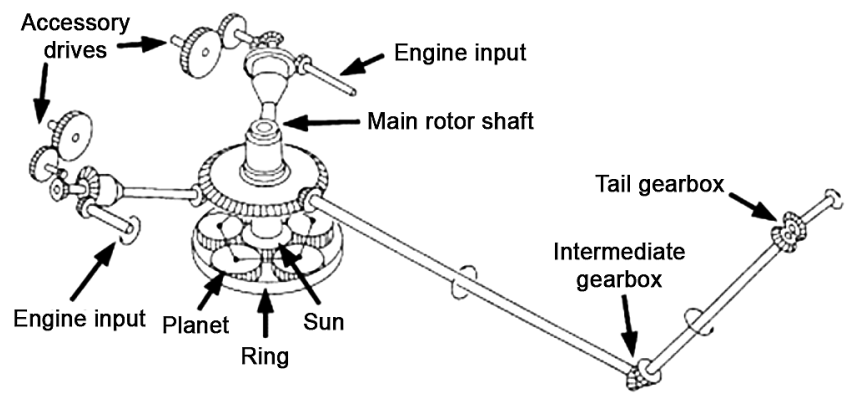

Figure 1. UH-60A planetary gear train schematic ${ }^{[7]}$

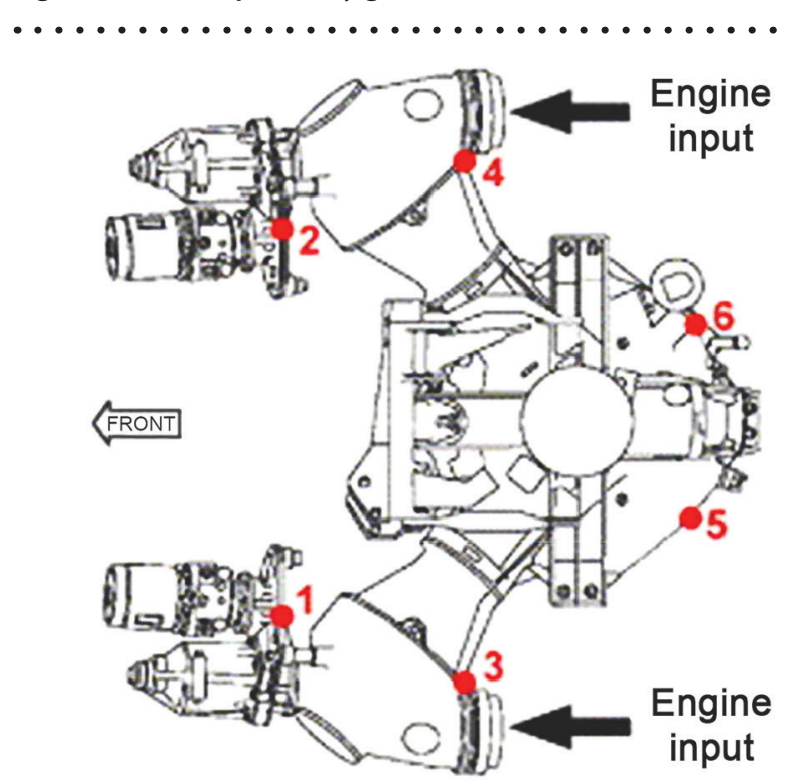

Figure 2. UH-60A transmission and sensor locations ${ }^{[7]}$ to analyse, one tool that can be used for the health assessment of planetary gear trains is vibration. The difficulty of assessing the health of planetary gear trains using vibration arises from many factors, such as the similar vibrations produced from multiple planet gears and the various time-varying transmission paths from gear mesh points to the sensors that are mounted on the gearbox housing. Thus, conventional fault detection algorithms may not be as effective when applied to such complicated gear trains.

In 2002, the main transmissions (2400 series) of two US Army UH-60A Black Hawk helicopters were found to contain fatigue cracks in the planet carriers, which are shown in Figures 3 and 4. When investigating the cause of repeated low transmission oil pressure warnings, a $250 \mathrm{~mm}$ hub-to-rim crack in the first carrier was found. An $82 \mathrm{~mm}$ crack in the second carrier was found during an inspection. These discoveries resulted in flight restrictions on many US Army UH-60A helicopters and an investigation into costeffective tests that were capable of diagnosing this type of fault.

A vibration test programme was conducted by the US Army on the transmission containing the $82 \mathrm{~mm}$ crack. The transmission was installed in a test-cell at various torque settings and was also groundrun in a helicopter at $20 \%$ and $30 \%$ torque settings. In addition, vibration data was acquired from undamaged transmissions.

The vibration data was first analysed in ${ }^{[1]}$ by applying planetary gearbox diagnostics to the time synchronous averages (TSAs) of the data. In this paper, the epicyclic sideband index (SI) and the epicyclic level factor (SLF) were found to effectively detect the presence of the fault using test-cell data, though neither were effective for the low-torque on-aircraft conditions. The same data was investigated by Dong et $a^{[2]}$ using a combination of wavelet analysis and Markov modelling. This method was able to distinguish the faulty component data from the healthy component data, though training of the datasets was required. Wu et al ${ }^{[3,4,5]}$ presented crack detection results via frequency and wavelet domain processing of the raw data that were reported to be dependent on the location of the sensor

- Submitted 22.05.15 / Accepted 14.09.15

Brandon Van Hecke, Jae Yoon and David He* are with the Department of Mechanical and Industrial Engineering, The University of Illinois at Chicago, 2039 ERF, 842 W Taylor Street, Chicago, IL 60607, USA.

${ }^{*}$ Corresponding author. 


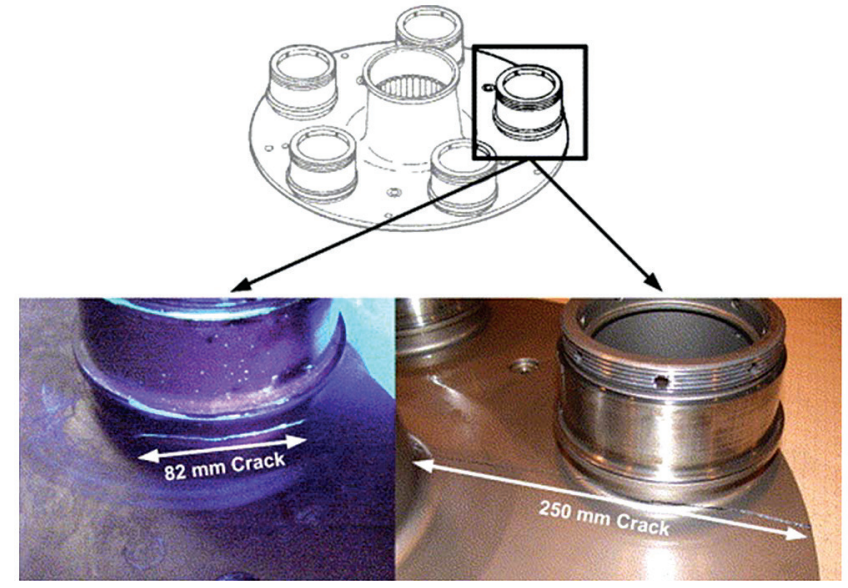

Figure 3. UH-60A planet carrier $82 \mathrm{~mm}$ crack (left) and $250 \mathrm{~mm}$ crack (right) ${ }^{[7]}$

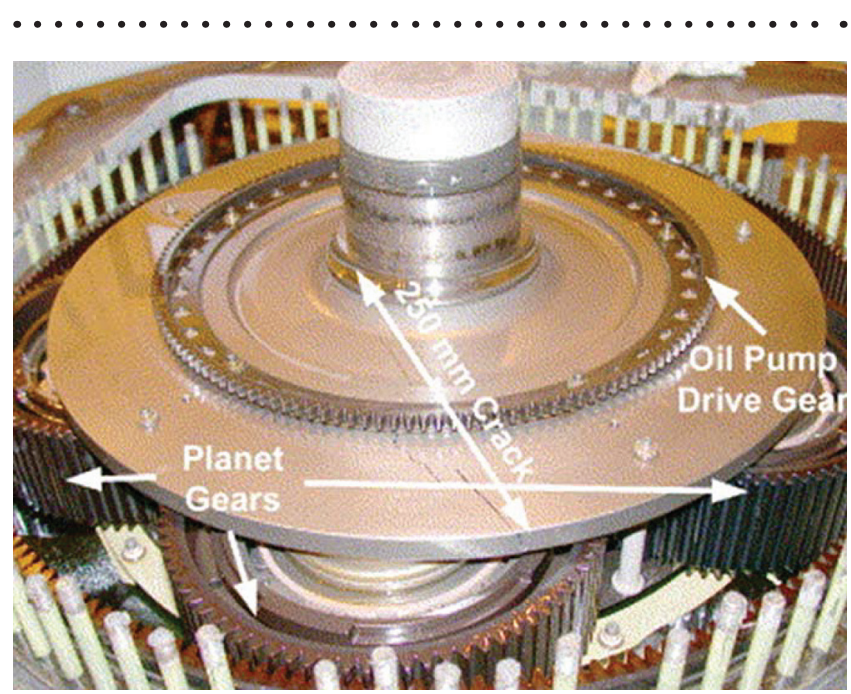

Figure 4. UH-60A planet carrier $250 \mathrm{~mm}$ crack (bottom view) ${ }^{[7]}$

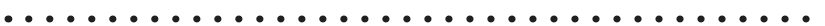

and frequency band. Mclnerny et $a l^{[6]}$ processed the test-cell data similarly to ${ }^{[1]}$ by applying a number of metrics to the TSAs of the data. In this paper, statistics were also presented for the raw data and a new metric measuring the ratio in the planet carrier TSA at multiples of planet-pass frequency with the remaining energy in the average was developed. Mclnerny et al ${ }^{[6]}$ were able to detect the crack in the test-cell, though did not investigate their metrics to the on-aircraft data. $\operatorname{In}^{[7]}$, two new methods of detecting the fatigue crack were developed. These methods were based on changes to the modulation of the fundamental gear mesh vibration resulting from the crack. These methods were again found to be effective for the test-cell conditions as reported in $^{[1]}$ and $^{[6]}$, though ineffective for the on-aircraft conditions.

The results presented in ${ }^{[1-7]}$ provided the motivation to develop a technique that can effectively detect fatigue cracks in on-aircraft conditions that would not require the need to train the datasets. This paper analyses the UH-60A on-aircraft vibration data using a time synchronous resampling-based spectral average approach. This technique has been shown to be effective in diagnosing all four bearing fault types for shaft speeds from $30 \mathrm{~Hz}$ to $60 \mathrm{~Hz}$, using both vibration and acoustic emission signals ${ }^{[8-10]}$. However, the time domain signal and statistical feature combination that was found to be effective for the aforementioned bearing applications was not effective for the data used in this study. It was found that the combination of a new analysis signal and condition indicators were effective for the evaluated UH-60A on-aircraft and test-cell vibration data. The results presented in this paper indicate that using such an approach can effectively detect the fatigue crack on the on-aircraft data using a number of condition indicators.

The remainder of this paper is structured as follows. Section 2 provides a detailed explanation of the methodology. In Section 3, the US Army UH-60A vibration data used to validate the methodology are discussed. Section 4 presents the planet carrier fatigue crack results and Section 5 concludes the paper.

\section{The methodology}

Figure 5 depicts an overview of the presented methodology. Firstly, the US Army Vibration Management Enhancement Program (VMEP) system is used to acquire the raw on-aircraft vibration data. Secondly, the vibration signal is time synchronously resampled using the shaft zero crossing times. Lastly, the resampled vibration signal is spectrally averaged and used to compute CIs for planet gear fatigue crack detection.

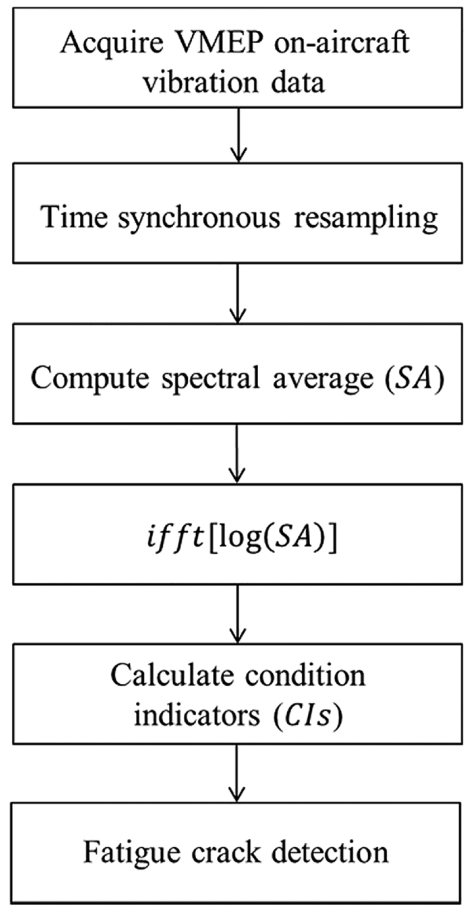

Figure 5. Overview of the methodology

The methodology will be presented in two sections. Section 2.1 provides a brief review of the time synchronous average (TSA), time synchronous resampling (TSR) and the spectral averaging approaches. Then, the calculation of condition indicators for planet carrier fatigue crack detection is explained in Section 2.2.

\subsection{Spectral averaging of the VMEP vibration signal}

TSA is a validated approach for the extraction of periodic waveforms and has multiple applications to the tooth meshing vibrations for gear fault diagnosis ${ }^{[11-16]}$. Moreover, TSA has been effectively utilised in the processing of vibration signal envelopes for bearing fault diagnosis ${ }^{[17-19]}$. The concept is to compute the ensemble average of sequential periods of any waveform of interest. This results in a significant reduction of noise and an enhanced signal that represents one period of the averaged waveform. Braun ${ }^{[20]}$ formally 
expressed the TSA $y(n T)$ of a signal $x(t)$ sampled at interval $n T$ as:

$$
y(n T)=(1 / N) \sum_{r=0}^{N-1} x(n T-r m T) .
$$

where the period being averaged is denoted by $m T$. Additional details concerning TSA can be found in ${ }^{[11]}$.

$\mathrm{In}^{[21]}$, it is shown that by using a synchronous resampling technique based on shaft revolutions, potential fluctuations in shaft speed can be accounted for and the effect of spectral smearing reduced. By resampling to an even number of points between trigger signal revolutions, a better fast Fourier transform (FFT) result was obtained. In this paper, a similar time synchronous resampling approach is accomplished using the shaft zero crossing times (ZCTs) and is formally presented in the following.

Formally, the resampling process is achieved by interpolating the $r$ number of data points in one shaft revolution into $L$ number of data points, such that:

$$
L=2^{\operatorname{Ceiling}[\log 2(r)]}
$$

where $L$ is the number of interpolated points between ZCTs and $r$ is the average number of points between shaft crossings before resampling. Once $L$ is determined, each segment contains an equal number of data points for FFT computations and the spectral average can be executed.

The methodology presented in this paper combines the TSR approach with spectral averaging, which allows for the extraction of effective condition indicators for planet carrier fatigue crack detection. Presented in Figure 6 is an overview of the spectral averaging approach. The computation of the spectral average requires the data to be sectioned so that the Fourier transform of each section can be computed. Then, the ensemble average of the squared magnitude of the segments is implemented.

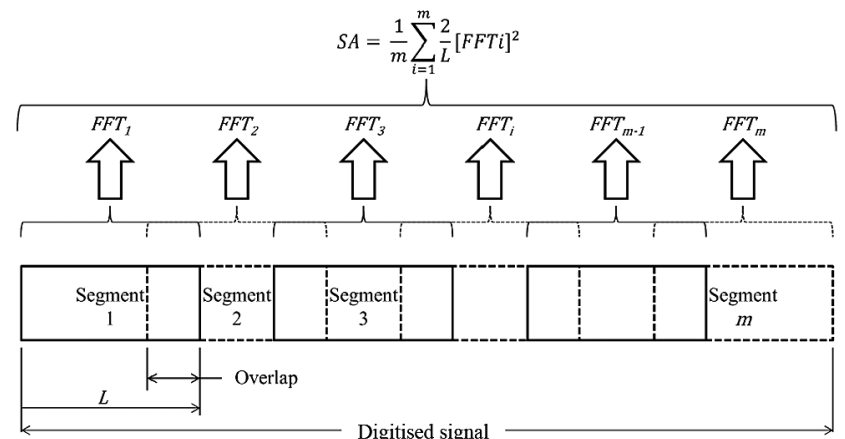

Figure 6. The spectral averaging approach

Welch $^{[22]}$ formally expressed the execution of this approach. By letting $Z(j)$, for $j=0, \ldots, N-1$ be a sample from a stationary, stochastic sequence whose mean is zero, then $Z(j)$ has a spectral density $P(f),|f| \leq \frac{1}{2}$, where $f$ is the normalised frequency. If one takes several possibly overlapping segments of length $L$, with the starting points of the segments $M$ points apart, and let $Z_{1}(j)$, $j=0, \ldots, L-1$ be the first segment, then:

$$
Z_{1}(j)=Z(j), \text { for } j=0, \ldots, L-1
$$

Likewise:

$$
Z_{2}(j)=Z(j+M), \text { for } j=0, \ldots, L-1
$$

and finally:

$$
Z_{K}(j)=Z(j+(K-1) M), \text { for } j=0, \ldots, L-1
$$

The result is $K$ segments, $Z_{1}(j), \ldots, Z_{K}(j)$, that cover the entire data sample $Z(j)$. Moreover, $(K-1) M+L=N$.

Next, for each segment of length $L$ the Fourier transform is obtained. In other words, a data window $W(j)$ is chosen, for $j=0, \ldots, L-1$, and the Fourier transforms $F_{1}(n), \ldots, F_{K}(n)$ of the segments $Z_{1}(j) W(j), \ldots, Z_{K}(j) W(j)$ are taken. This is formally expressed in Equation (6):

$$
F_{k}(n)=\frac{1}{L} \sum_{j=0}^{L-1} Z_{k}(j) W(j) e^{-2 k i j n / L}
$$

where $i=(-1)^{\frac{1}{2}}$. The result is $K$ Fourier transforms $B_{k}\left(f_{n}\right)$, which correspond to the $K$ number of segments:

$$
B_{k}\left(f_{n}\right)=\frac{L}{S}\left|F_{k}(n)\right|^{2}, \text { for } k=1,2, \ldots, K
$$

where:

and:

$$
f_{n}=\frac{n}{L}, \text { for } n=0, \ldots, L / 2
$$

$$
S=\frac{1}{L} \sum_{j=0}^{L-1} W^{2}(j)
$$

Last, the spectral average result, $S A\left(f_{n}\right)$, is obtained by taking the average of the $K$ Fourier transforms:

$$
S A\left(f_{n}\right)=\frac{1}{K} \sum_{k=1}^{K} B_{k}\left(f_{n}\right)
$$

To implement the spectral average, the signal must first be sectioned. Thus, the shaft ZCTs are used to section and resample the vibration signal. Then, the average of the squared magnitude spectrum of the sections is obtained, which results in the spectral average used to evaluate statistical features. In other words, the duration between shaft revolutions is utilised as the sectioning function to average the UH-60A VMEP vibration signal. Additionally, the data segment length $L$ is determined by the number of data points between ZCTs of the shaft after resampling. By resampling the segments to $L$ data points, an equal number of data points are used for FFT calculations. Hence, the approach presented in this paper utilises the ZCTs of shaft rotations to section and resample the vibration signals and compute the ensemble average of the squared magnitude Fourier transforms of the sections. This approach affords the ability to compute a spectral average and effectively detect a planet carrier fatigue crack. After computing the spectral average of the vibration signals, various fatigue crack features are computed and evaluated as condition indictors.

\subsection{Condition indicators for planet carrier fatigue crack detection}

There are numerous condition indicators in literature that quantify accelerometer signals to aid in rotating machinery fault diagnosis. For example, some recent studies have developed effective CIs that accomplish bearing fault diagnosis by quantifying both accelerometer and $\mathrm{AE}$ signals ${ }^{[8-10,23-24]}$. A major difference among the available CIs is in the method of computation. For example, CIs can be extracted from both the time and frequency domains of the signals. Furthermore, CIs can be extracted from a raw signal or a signal being processed by a signal processing technique, such as TSA or spectral averaging. Previously, the inverse Fourier transform of the square magnitude of the spectral average in combination with the RMS and peak CIs has been used to successfully diagnose bearing faults at shaft speeds of $30 \mathrm{~Hz}$ and higher for both $\mathrm{AE}$ and vibration signals ${ }^{[8-10]}$. However, because the aforementioned analysis signal and CI combination tested in those studies were not effective for shaft speeds less than $30 \mathrm{~Hz}$, such work was left for future investigation. This paper uses the inverse Fourier transform of the 
$\log$ of the squared magnitude spectral average result to investigate the potential of CIs that allow detection of the planet carrier fatigue crack in on-aircraft conditions. By introducing a log into the inverse Fourier transform, the signal being analysed is similar to a power cepstrum that has been utilised in ${ }^{[25]}$ for gear analysis. It should also be mentioned that although the introduction of the log was found to be effective for the UH-60A application, it was not effective for the high-speed bearing data used in ${ }^{[8-10]}$. Formally, the input signal used for CI computation is given as:

$$
x=\text { ifft }\left\{\log \left[S A\left(f_{n}\right)\right]\right\}
$$

where the input signal $x$ is the time domain signal used for CI computation and ifft $\left\{\log \left[S A\left(f_{n}\right)\right]\right\}$ is the inverse Fourier transform of the log of the spectral average result $S A\left(f_{n}\right)$ obtained by Equation (10).

CIs can be computed using an energy operator (EO). The EO is a type of residual of the autocorrelation function ${ }^{[26]}$. In discretised form, the mathematical formula is given as:

$$
E O\left[x_{i}\right]=x_{i}^{2}-x_{i-1} \cdot x_{i+1}
$$

where $E O\left[x_{i}\right]$ is the $i$ th element in the $\mathrm{EO}$ and $x_{i}$ is the $i$ th element of dataset $x$ obtained from Equation (11).

The CIs are also computed using the amplitude modulation (AM) signal or Hilbert envelope of the signal obtained in Equation (11). The AM is formally obtained by the following:

$$
A M=a b s[\operatorname{hilbert}(x)] \text {. }
$$

where abs[hilbert $(x)]$ is the Hilbert envelope of the dataset $x$ acquired using Equation (11).
In this paper, a number of feasible CIs were explored for planet carrier fatigue crack detection. Provided in Table 1 are the definitions of the investigated CIs. Presented are the definitions for root mean square (RMS), peak, crest factor $(C F)$, kurtosis (Kurt), skewness (Skew), peak-to-peak $(p 2 p)$ and histogram upper bound $(U B)$. Each $\mathrm{CI}$ is evaluated on the signals computed using Equations (11), (12) and (13).

\section{UH-60A on-aircraft vibration data}

The test-cell experiments were conducted in the Helicopter Transmission Test Facility (HTTF) at the Patuxent River Naval Air Station in Maryland, USA. The transmission with the $82 \mathrm{~mm}$ planet carrier crack was tested along with a healthy transmission. A total of six accelerometers and two tachometers were used for data acquisition. Raw time domain data was collected over a range of torque levels at a sampling rate of $100 \mathrm{kHz}$, for a length of $180 \mathrm{~s}$.

The on-aircraft vibration data was acquired using the US Army VMEP system ${ }^{[27-29]}$. At the Corpus Christ Army Depot (CCAD), the transmission with the $82 \mathrm{~mm}$ cracked carrier was installed and tested in a UH-60A helicopter (aircraft X). Three unfaulted UH-60A helicopters (aircrafts A, B and C) were also tested from the Birmingham, Alabama, National Guard (BNG). For safety precautions, only ground runs at $20 \%$ and $30 \%$ torque settings were completed and each aircraft was stabilised for $5 \mathrm{~min}$ at each state before vibration acquisitions were completed. For all vibration measurements, the nose was pointed in the direction of the wind, which was less than 10 knots. It is also important to note that the

\begin{tabular}{|c|c|c|c|c|}
\hline \multirow[b]{2}{*}{$\mathrm{CI}$} & \multirow[b]{2}{*}{ Equation Description } & \multicolumn{3}{|c|}{ Signal $y$ used to compute the CIs } \\
\hline & & Input signal $(x)$ & EO of input signal $(x)$ & AM of input signal $(x)$ \\
\hline Root mean square $(R M S)$ & $\sqrt{\left(\frac{1}{N}\right) \sum_{i=1}^{N} y_{i}^{2}}$ & \multicolumn{3}{|c|}{ RMS: statistical measure of the magnitude of a varying quantity } \\
\hline Peak & $\frac{\left(y_{\max }-y_{\min }\right)}{2}$ or $y_{\max }$ & \multicolumn{3}{|c|}{ Peak: maximum value in the dataset } \\
\hline Crest factor $(C F)$ & $\frac{y_{\text {peak }}}{y_{\text {rms }}}$ & \multicolumn{3}{|c|}{$C F$ : the ratio of peak to RMS; describes how extreme the peaks are in a waveform } \\
\hline Kurtosis (Kurt) & $\frac{N \sum_{i=1}^{N}\left(y_{i}-\bar{y}\right)^{4}}{\left[\sum_{i=1}^{N}\left(y_{i}-\bar{y}\right)^{2}\right]^{2}}$ & \multicolumn{3}{|c|}{ Kurt: describes the peakedness or smoothness of the dataset } \\
\hline Skewness (Skew) & $\frac{\left(\frac{1}{N}\right) \sum_{i=1}^{N}\left(y_{i}-\bar{y}\right)^{3}}{\left[\sqrt{\left(\frac{1}{N}\right)} \sum_{i=1}^{N}\left(y_{i}-\bar{y}\right)^{2}\right]^{3}}$ & \multicolumn{3}{|c|}{$\begin{array}{l}\text { Skew: measures the asymmetry of the data around its sample mean. A negative or } \\
\text { positive value of skew implies the data is spread to the left or right of the mean, } \\
\text { respectively }\end{array}$} \\
\hline Peak-to-peak $(p 2 p)$ & $\left(y_{\max }-y_{\min }\right)$ & \multicolumn{3}{|c|}{$\begin{array}{c}p 2 p: \text { measures the distance between the maximum and minimum value in the } \\
\text { dataset }\end{array}$} \\
\hline Histogram upper bound $(U B)$ & $y_{\max }+\frac{1}{2}\left[\frac{\left(y_{\max }-y_{\min }\right)}{(N-1)}\right]$ & \multicolumn{3}{|c|}{$U B$ : highest frequency bin value in the dataset } \\
\hline
\end{tabular}
cyclic stick position may have varied between acquisitions.

\section{Table 1. The definitions of the Cls}


The raw data was only acquired for aircrafts A and X using the VMEP system. Additionally, only accelerometers 3 and 5 from Figure 2 were recorded. The sampling rate for the raw data was $48 \mathrm{kHz}$ with a bandwidth of $18.75 \mathrm{kHz}$. The acquisition lengths were $25 \mathrm{~s}$, equating to data records of 1.2 million samples per acquisition. Further information regarding planetary gear mesh vibration can be found in ${ }^{[7]}$.

\section{Results}

Firstly, the results for the test-cell data will be discussed. Of all the loading conditions, only the $20 \%$ torque test-cell data for both transmissions was available for analysis. Thus, since only one acquisition of $180 \mathrm{~s}$ was available for each transmission, the data was segmented into $30 \mathrm{~s}$ intervals to provide more samples while ensuring enough tachometer revolutions for spectral average computations. The result was six segments of $30 \mathrm{~s}$ for each transmission at the aforementioned torque level and the methodology presented in Figure 5 was implemented. Additionally, since sensor 5 was closest to the source, it was the only sensor investigated. After the CIs were computed, $95 \%$ confidence intervals were obtained to determine the statistical significance of the CI values. It was found that four CIs clearly differentiated the $82 \mathrm{~mm}$ crack transmission from the healthy transmission. Figure 7 presents the average CI values for the six samples along with the $95 \%$ confidence intervals for sensor 5 test-cell data.

Next, the results of the UH-60A on-aircraft VMEP data analysis are presented. All VMEP acquisitions were processed following the methodology presented in Figure 5. To determine if the fatigue crack was detectable for the on-aircraft data using the presented approach, the two sample CI values at each torque setting were averaged. For sensor 3, the average CI values for each torque level are presented in Table 2. Once the average CI values were computed, the percent difference in the CI values between aircraft $\mathrm{X}$ and aircraft $\mathrm{A}$ was calculated. For sensor 3, it was found that the four aircraft X CI means were separable from aircraft A CI means

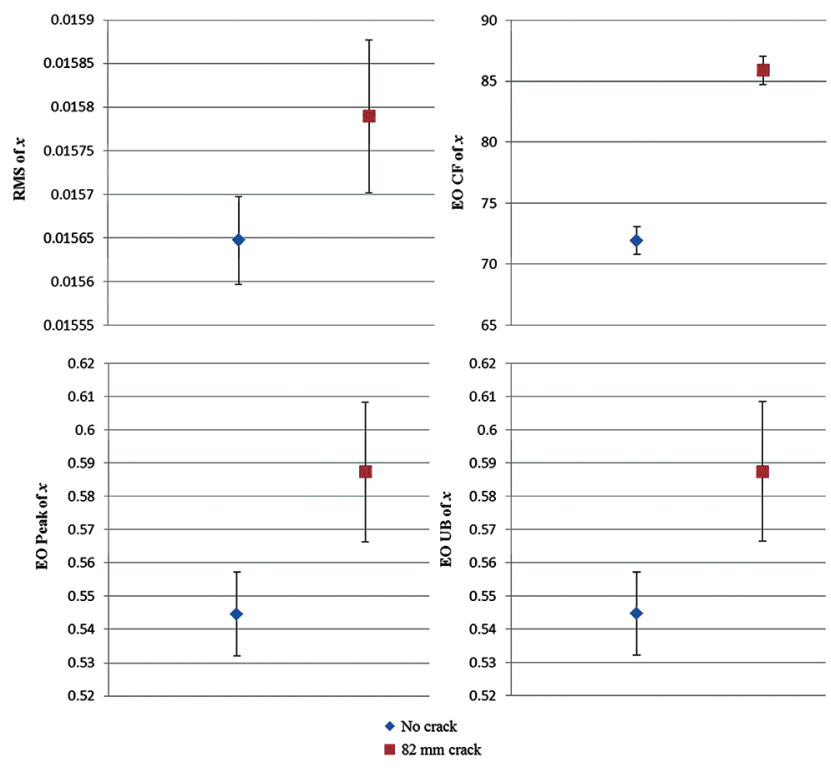

Figure 7. Average $\mathrm{Cl}$ values with $95 \%$ confidence interval for sensor 5 test-cell data

Insight • Vol 57 • No 11 • November 2015 for both torque settings of $20 \%$ and $30 \%$. Then, to examine which CIs performed better in terms of the separation, they were ranked in Table 2 from the highest to the lowest percent difference. It was found that the order of the CI ranking was the same for both torque settings, which confirms the torque independence of the CI values. For sensor 3, the best CI was EO peak with percent differences of 86.794 and 105.375 for $20 \%$ and $30 \%$ torque, respectively. Figure 8 presents the average EO peak CI values at the $20 \%$ and $30 \%$ torque settings for both aircraft $\mathrm{X}$ and aircraft $\mathrm{A}$.
Table 2. Sensor $3 \mathrm{Cl}$ average results

\begin{tabular}{|c|c|c|c|c|c|}
\multicolumn{7}{|c|}{ Sensor 3 } \\
\hline Aircraft X & Aircraft A & & Aircraft X & Aircraft A & \\
\hline $20 \%$ torque setting & \% difference & \multicolumn{2}{|c|}{$30 \%$ torque setting } & \% difference \\
\hline 5.348 & 2.863 & 86.794 & 5.210 & 2.537 & 105.3745 \\
\hline 5.348 & 2.863 & 86.793 & 5.210 & 2.537 & 105.3728 \\
\hline 0.030 & 0.026 & 17.094 & 0.029 & 0.024 & 19.997 \\
\hline 90.055 & 87.637 & 2.758 & 90.022 & 86.632 & 3.912 \\
\hline
\end{tabular}

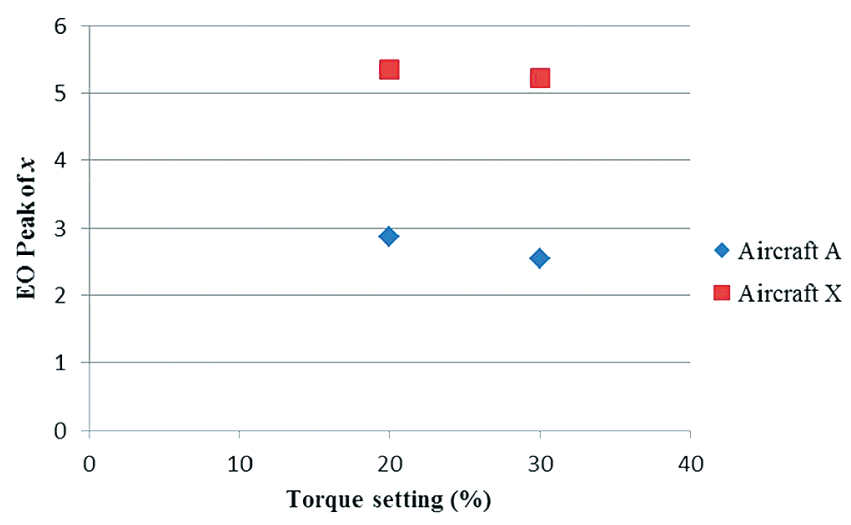

Figure 8. Average EO peak $\mathrm{Cl}$ by torque setting for sensor 3

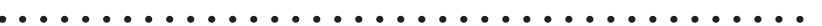

The VMEP acquisitions for sensor 5 were processed in the same manner as sensor 3 . Thus, the CI values at each torque level were averaged and the percent difference between aircraft $\mathrm{X}$ and aircraft A computed. The results for all CIs are presented in Table 3. Similar to the sensor 3 results, the CI rankings were the same for both torque settings, which again confirms the torque independence of the CI values. Also, the best CI for sensor 5 was EO peak. The average EO peak CI results are shown at both torque settings for both aircraft $\mathrm{X}$ and aircraft $\mathrm{A}$ in Figure 9. It is important to note that the top two CIs for sensor 5 perform better than the top CI (EO peak) for sensor 3 in terms of percent difference. The reason for the higher percent difference using sensor 5 data is likely to be due to the fact that sensor 5 was much closer to the planet carrier fatigue crack. The close proximity of sensor 5 to the fatigue crack location induces an increase in the acceleration of displacement. As a result, the amplitude of the sensor output is raised and is accompanied by an excited response and behaviour of the signal. Thus, the peak value extracted from a sensor closer to the fault location is going to be larger than for sensors further away. This phenomena is why the EO peak value is larger when computed from a sensor attached to the fault location versus a sensor attached to a nearby component. Similarly, an increase in acceleration results in a higher extremeness of the peak value in the signal and hence a larger EO CF result. Based on this reasoning, it can also be understood why the energy in the signal (RMS) and EO UB values are larger for sensor 5, which 
Table 3. Sensor $5 \mathrm{Cl}$ average results

\begin{tabular}{|c|c|c|c|c|c|c|}
\hline & \multicolumn{7}{c|}{ Sensor 5 } \\
\cline { 2 - 8 } & Aircraft X & Aircraft A & & Aircraft X & Aircraft A & \\
\hline CI & $20 \%$ torque setting & \% difference & $30 \%$ torque setting & \% difference \\
\hline EO Peak & 3.121 & 1.083 & 188.246 & 3.021 & 1.013 & 198.265 \\
\hline EO UB & 5.316 & 1.961 & 171.112 & 5.127 & 1.848 & 177.524 \\
\hline RMS & 0.030 & 0.025 & 19.489 & 0.029 & 0.025 & 16.353 \\
\hline EO CF & 51.556 & 49.045 & 5.119 & 51.646 & 48.360 & 6.795 \\
\hline
\end{tabular}

were processed. For each sensor, two samples of data were analysed for both the $20 \%$ and $30 \%$ torque settings. The results indicate that using the presented approach, a total of four CIs can be used to detect the fatigue crack in a planet carrier for both the test-cell and the on-aircraft UH-60A vibration data. The CIs were ranked in the order of percent difference of aircraft X mean CI values from aircraft A mean CI values. The ranking for both sensor data was constant regardless of torque setting, indicating that the CIs are torque

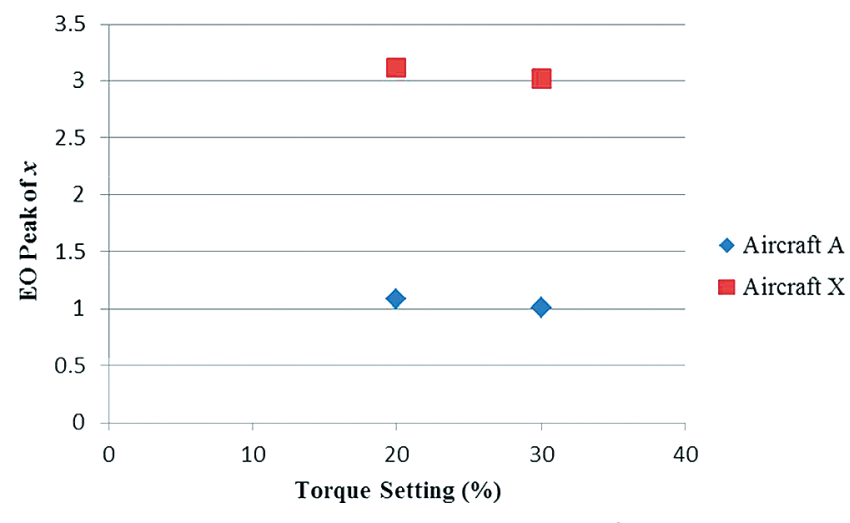

Figure 9. Average EO peak $\mathrm{Cl}$ by torque setting for sensor 5

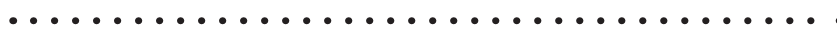

is local to the source of the damage.

The presented methodology has been validated to detect the single planet gear carrier fatigue crack using the test-cell and on-aircraft data described in the paper. The US Army initiative began as a result of single fatigue cracks observed during inspection. The presence of a crack on the planet gear carrier excites a response from the accelerometer, which increases the peak amplitude and overall energy in the signal. The change in behaviour in the signal is then extracted using the CIs tested in this paper. With additional data, healthy planet gear carrier CI threshold values could be set. Any CI values exceeding the set threshold would be indicative of the presence of a fault. If more than one crack is in the system, the increase in CI values would be picked up, since the SA waveform is synchronised with the main rotor tachometer signal. In other words, the enhanced synchronous waveform will contain abnormal periodic components that are extracted using the tested CIs and therefore differentiated from the healthy state. Thus, this methodology is designed to detect damage that excites periodic components that are synchronous with the main rotor shaft.

\section{Conclusions}

In this paper, a new method for detecting an on-aircraft fatigue crack in a planet carrier of an epicyclic transmission was presented. This method begins by time synchronously resampling vibration signals to segment the signals according to shaft crossing times, such that an even number of data points are obtained to compute a spectral average. Then, the result is used to extract features and evaluate numerous CIs for planet carrier fatigue crack detection. The methodology was validated using the raw vibration test-cell and on-aircraft data acquired from a number of US Army UH-60A Black Hawk helicopter main transmissions. For the test-cell data, analysis was carried out for sensor 5 at the $20 \%$ torque setting. For the on-aircraft data, both sensor 3 and sensor 5 acquisitions independent. Also, the EO peak CI was the best for both sensors in terms of percent difference. The studies currently reported in the literature have had difficulty detecting the fatigue crack using the on-aircraft data. The results in this paper indicate that the presented approach can be utilised to address the detection of fatigue cracks in a planet carrier of a UH-60A helicopter using VMEP vibration data.

The presented approach was designed to detect any abnormalities on the planet gear carrier. The damage is detected by extracting the CIs from the enhanced signal that was synchronised with the main rotor shaft. Because the approach was designed to detect any irregularities, it should theoretically capture defects that are of different directions, orientations or depths than those tested in the US Army experiments. In fact, the separation of the faulty CI values from the healthy CI values will be more significant for the case of a larger crack depth or width. On that note, accelerometers measure the second derivative of displacement, so the orientation of the sensor is critical. One drawback would be the case where damage does not excite any response due to the sensor placement. However, through the combination of the correct positioning of the sensors with the periodic excitement of the signal due to rotational passes of the crack, any damage on the planet carrier will be detected and differentiated from a healthy transmission case.

\section{References}

1. J A Keller and P Grabill, 'Vibration monitoring of UH-60A main transmission planetary carrier fault', American Helicopter Society 59th Annual Forum, Phoenix, Arizona, USA, 6-8 May 2003.

2. M Dong, D He, P Banerjee and J A Keller, 'Equipment health diagnosis and prognosis using advanced hidden Markov models', 58th Meeting of the Machinery Failure Prevention Technology Society, Virginia Beach, Virginia, USA, 26-30 April 2004.

3. B Wu, A Saxena, T S Khawaja, R Patrick, G Vachtsevanos and P Sparis, 'An approach to fault diagnosis of helicopter planetary gears', IEEE Autotestcon, San Antonio, Texas, USA, 20-23 September 2004.

4. B Wu, A Saxena, R Patrick and G Vachtsevanos, 'Vibration monitoring for fault diagnosis of helicopter planetary gears', 16th IFAC World Congress, Prague, Czech Republic, 3-8 July 2005.

5. A Saxena, B Wu and G Vachtsevanos, 'A methodology for analysing vibration data from planetary gear systems using complex Mortlet wavelets', American Control Conference, Portland, Oregon, USA, 8-10 June 2005.

6. S A McInerny, W Hardman, J A Keller and R Bednarczyk, 'Detection of a cracked planet carrier', 10th International Congress on Sound and Vibration, Stockholm, Sweden, 7-10 July 2003.

7. D M Blunt and J A Keller, 'Detection of a fatigue crack in a UH-60A planet gear carrier using vibration analysis', Mechanical Systems and Signal Processing, Vol 20, No 8, pp 
2095-2111, 2006.

8. B Van Hecke, D He and Y Qu, 'On the use of spectral averaging of acoustic emission signals for bearing fault diagnostics', ASME Journal of Vibration and Acoustics, 2014. DOI: $10.1115 / 1.4028322$

9. B Van Hecke, Y Qu and D He, 'Bearing fault diagnosis based on a new acoustic emission sensor technique', Proceedings of the Institution of Mechanical Engineers, Part O: Journal of Risk and Reliability, 2014. DOI: 10.1177/1748006X14558900

10. B Van Hecke, Y Qu, D He and E Bechhoefer, 'A new spectral average-based bearing fault diagnostic approach', Journal of Failure Analysis and Prevention, Vol 14, No 3, pp 354-362, 2014.

11. P D McFadden, 'A revised model for the extraction of periodic waveforms by time domain averaging', Mechanical Systems and Signal Processing, Vol 1, No 1, pp 83-95, 1987.

12. P D McFadden, 'A technique for calculating the time domain averages of the vibration of the individual planet gears and the sun gear in an epicyclic gearbox', Journal of Sound and Vibration, Vol 144, No 1, pp 163-172, 1991.

13. F Bonnardot, M El Badaoui, R B Randall, J Daniere and F Guillet, 'Use of the acceleration signal of a gearbox in order to perform angular resampling (with limited speed fluctuation), Mechanical Systems and Signal Processing, Vol 19, No 4, pp 766-785, 2005.

14. E Bechhoefer, 'An enhanced time synchronous averaging for rotating equipment analysis', Proceedings for the Joint Conference: Machinery Failure Prevention Technology 2013 and International Instrumentation Symposium 2013, Cleveland, Ohio, USA, pp 149-158, 13-17 May 2013.

15. Y Qu, E Bechhoefer, D He and J Zhu, 'A new acoustic emission sensor-based gear fault detection approach', International Journal of Prognostics and Health Management, Vol 4, No 2, pp 1-14, 2013.

16. Y Qu, J Zhu, D He, B Qiu and E Bechhoefer, 'Time synchronous average-based acoustic emission signal analysis on gear fault detection', IEEE International Conference on Prognostics and Health Management, Gaithersburg, Maryland, USA, 24-27 June 2013

17. P D McFadden and M M Toozhy, 'Application of synchronous averaging to vibration monitoring of rolling element bearings', Mechanical Systems and Signal Processing, Vol 14, No 6, pp 891-906, 2000.

18. D Siegel, H Al-Atat, V Shauche, L Liao, J Snyder and J Lee, 'Novel method for rolling element bearing health assessment - a tachometer-less synchronously-averaged envelope feature extraction technique', Mechanical Systems and Signal Processing, Vol 29, No 1, pp 362-376, 2012.

19. K N Christian, N Mureithi, A Lakis and M Thomas, 'On the use of synchronous averaging, independent component analysis and support vector machines for bearing fault diagnosis', First International Conference on Industrial Risk Engineering, Montreal, Québec, Canada, pp 610-624, 17-19 December 2007.

20. S Braun, 'The extraction of periodic waveforms by time domain averaging, Acustica, Vol 32, pp 69 -77, 1975.

21. E Bechhoefer, B Van Hecke and D He, 'Processing for improved spectral analysis', Annual Conference of the Prognostics and Health Management Society, New Orleans, Louisiana, USA, pp 14-17, 14-17 October 2013.

22. P Welch, 'The use of fast Fourier transform for the estimation of power spectra: a method based on time averaging over short, modified periodograms', IEEE Transactions on Audio and Electroacoustics, Vol 15, No 2, pp 70-73, 1967.
23. D He, R Li, J Zhu and M Zade, 'Data mining-based full ceramic bearing fault diagnostic system using AE sensors', IEEE Transactions on Neural Networks, Vol 22, No 12, pp 20222031, 2011.

24. D He, R Li, M Zade and J Zhu, 'Development and evaluation of AE-based condition indicators for full ceramic bearing fault diagnosis', IEEE International Conference on Prognostics and Health Management, Denver, Colorado, USA, 20-23 June 2011.

25. M El Badaoui, J Antoni, F Guillet and J Daniere, 'Use of the moving cepstrum integral to detect and localise tooth spalls in gears', Mechanical Systems and Signal Processing, Vol 15, No 5, pp 873-885, 2001.

26. H M Teager and S M Teager, 'Evidence for non-linear sound production mechanisms in the vocal tract', Speech Production and Speech Modelling Symposium, Time Frequency and TimeScale Analysis, Victoria, British Columbia, Canada, pp 345348, 1992.

27. P Grabill, J Berry, L Grant and J Porter, 'Automated helicopter vibration diagnostics for the US army and national guard', American Helicopter Society 57th Annual Forum, Washington, DC, USA, 9-11 May 2001.

28. P Grabill, T Brotherton, J Berry and L Grant, 'The US army and national guard vibration management enhancement programme (VMEP): data analysis and statistical results', American Helicopter Society 58th Annual Forum, Montreal, Canada, 11-13 June 2001.

29. P Grabill, T Brotherton, J Berry, B Branhof and L Grant, 'Rotor smoothing and vibration monitoring results for the US army VMEP', American Helicopter Society 59th Annual Forum, Phoenix, Arizona, USA, 6-8 May 2003.

\section{(5)}

\section{For your specialist EMAT \&} ultrasonic needs in research and NDT

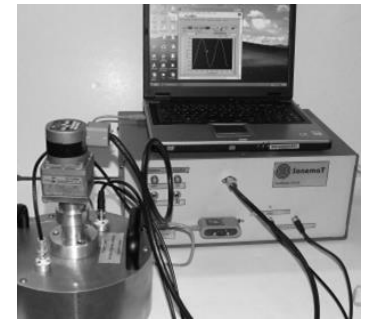

Advanced materials analysis equipment

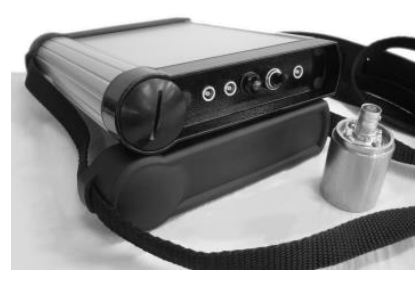

EMAT based flaw detection
Consultancy and custom work undertaken! Sonemat Ltd., The Venture Centre, Sir William Lyons Road, Coventry CV4 7EZ, phone 02476 574116, www.sonemat.co.uk

Authorised agent for 슈를 High-power ultrasonics 


\section{Non-Destructive Testing (NDT)}

\section{Engineering Technician Apprenticeship}

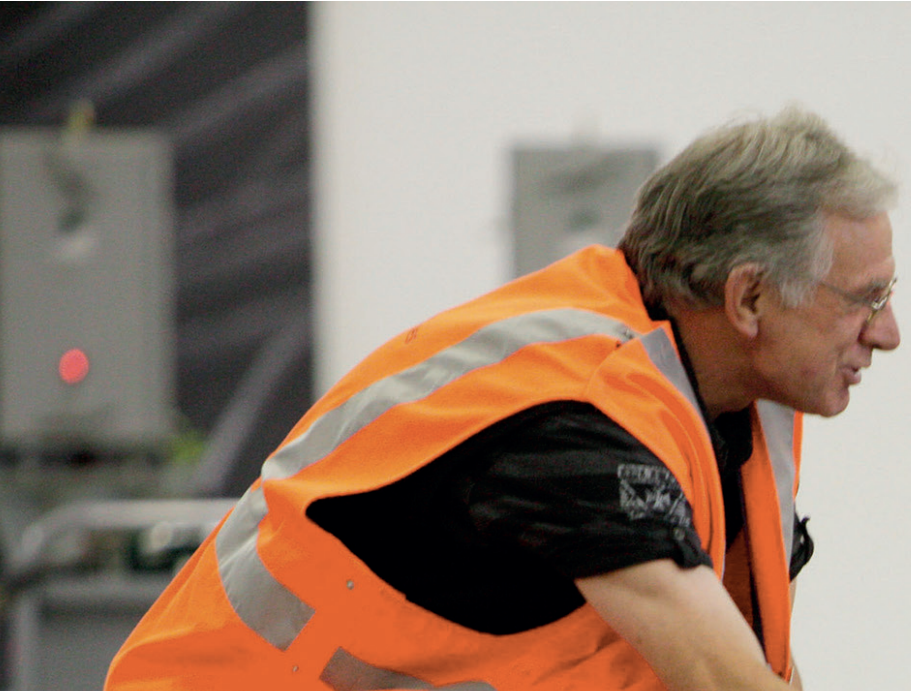

Secure funding to train and develop your NDT Apprentices

Apprenticeships bring fresh ideas and new thinking to your business

\section{www.bindt.org/apprenticeships}

Apprenticeships 
Copyright of Insight: Non-Destructive Testing \& Condition Monitoring is the property of British Institute of Non-Destructive Testing and its content may not be copied or emailed to multiple sites or posted to a listserv without the copyright holder's express written permission. However, users may print, download, or email articles for individual use. 